\title{
RED CELL DISTRIBUTION WIDTH AND PLATELET INDICES AS PREDICTORS IN DETERMINING THE PROGNOSIS OF UPPER GASTROINTESTINAL SYSTEM BLEEDING
}

\author{
Abaylı Bahri, ${ }^{1}$ Gencdal Genco ${ }^{2}$ \\ ${ }^{1}$ Cukurova Dr. Askım Tufekci Hospital, Department of Gastroenterology and Hepatology, Adana, Turkey \\ 2 Istanbul Atasehir Memorial Hospital, Department of Gastroenterology, Istanbul, Turkey
}

Primljen/Received 17. 04. 2019. god.

Prihvaćen/Accepted 17. 06. 2019. god.

\begin{abstract}
Background and Aim: Acute upper gastrointestinal bleeding (AUGIB) is one of the most common medical emergencies. Early detection of at-risk patients is beneficial with respect to treatment and prognosis. We investigated whether severity of ulcers were associated with red blood cell distribution width (RDW), plateletcrit (PCT), mean platelet volume (MPV) and platelet distribution width (PDW). Materials and Methods: All patients presenting to the emergency department with AUGIB between January 2014 and December 2017 were included in the study. Endoscopy reports, complete blood counts, patient demographic characteristics, and endoscopy results were obtained retrospectively from hospital records. Patients with grade I or grade II ulcers (based on the Forrest classification) were compared in regard to these parameters. Results: In total, 373 male and 211 female patients with a mean age \pm SD of $66.36 \pm 17.36$ were included in this study. Ulcers were detected in 396 of 584 patients $(67.8 \%)$. There were no differences with respect to RDW or platelet indices between groups.
\end{abstract}

Conclusions: Patients presenting with AUGIB should receive rapid diagnosis and treatment. In this study of patients with AUGIB grouped by bleeding aetiology, probable early prognostic parameters were not associated with bleeding severity among patients diagnosed with gastric or duodenal ulcers.

Key words: Endoscopy, gastrointestinal bleeding, ulcer, RDW, PCT, MPV, PDW.

\section{INTRODUCTION}

Acute upper gastrointestinal bleeding (AUGIB) usually manifests itself with hematemesis, melena, or both. Most cases of AUGIB resolve spontaneously $(70 \%-80 \%)$ and mortality is low $(3 \%-6 \%)$. However, patients should be rapidly diagnosed and treated. Erythrocyte detection in nasogastric lavage at admission, tachycardia, and haemoglobin level less than 8 $\mathrm{g} / \mathrm{dl}$ indicate severe AUGIB. Early detection of high-risk patients affects prognosis and treatment and is cost-effective (1-7).

Red cell distribution width (RDW), a measure of the variability in size of circulating erythrocytes, is used routinely in clinical practice to determine anaemia subtype. Studies also suggest that RDW predicts morbidity and mortality for various severe diseases. Furthermore, RDW may independently predict development of massive and recurrent bleeding in patients experiencing percutaneous coronary intervention, intracranial hematoma, or multiple traumas. Plateletcrit (PCT), mean platelet volume (MPV), and platelet distribution width (PDW) are routinely evaluated as part of the complete blood count. It has been suggested that these parameters may be useful in understanding inflammatory, ischemic, and thrombotic conditions as well. To date, the number of studies addressing the relationship between gastrointestinal diseases and platelet indices is low. A few studies have shown MPV to be low during acute exacerbations ofinflammatory bowel disease and MPV to be high among patients with colon cancer and a poor prognosis. Upper gastrointestinal endoscopy (UGE), which is usually required to diagnose and manage AUGIB in the hospital, plays an important role in determining AUGIB severity and aetiology. In patients with haemorrhage, a wide variety of endoscopic methods (eg, endoscopic sclerotherapy and endoscopic haemoclipping) are commonly used. The Forrest classification, which is commonly used in conjunction with UGE to describe peptic ulcers, isvery useful in determining re-bleeding risk; however, UGE is not 
performed routinely at all health centres. Patients presenting with severe AUGIB should be selected carefully for prompt transfer to centres with endoscopy capabilities (8-14).

In this study, our primary aim was to describe AUGIB aetiology for patients presenting to our hospital. Secondarily, we aimed to determine whether AUGIB severity (Forrest classification) was related to RDW, PCT, MPV, and PDW.

\section{MATERIALS AND METHODS}

Patient data and endoscopy reports were retrospectively obtained from hospital records. Between January 2014 and December 2017, patients who were presented to our emergency department with AUGIB and underwent upper gastrointestinal endoscopy were eligible for the inclusion in the study. Patients were excluded if they had a history of haematologic disease, refused laboratory testing or treatment, requested transfer to another facility, were pregnant, or had medical records that lacked clinical or laboratory data. Approval from local ethics committee and written/informed consent from patients were not obtained because the study was retrospective. The study conformed to the ethical guidelines of the 1975 Declaration of Helsinki.

Endoscopy reports for patients with confirmed gastric or duodenal ulcers were classified by a gastroenterologist according to the Forrest classification: 1a and $1 \mathrm{~b}$ ulcers were classified as high re-bleeding risk; $2 \mathrm{a}$, $2 \mathrm{~b}$ and $2 \mathrm{c}$ ulcers were classified as increased re-bleeding risk. Patients were divided into 4 groups according to RDW:(1) RDW less than 12.8; (2) RDW greater than 12.9 and less than 14.4 ; (3) RDW greater than 14.5 and less than 16.5; or (4) RDW greater than 16.6.

Complete blood count values and demographic characteristics were retrospectively obtained from hospital records. Patients with high re-bleeding risk (Forrest 1) and increased re-bleeding risk (Forrest 2) were compared to each other.

\section{RESULTS}

Demographic characteristics and endoscopy results for 584 patients $(63.9 \%$ male) were included in this study. The mean \pm SD age was $66.36 \pm 17.36$ years. Demographic characteristics and endoscopy results are presented in Table 1 and Figure 1. Duodenal and gastric ulcers were the most common and second most common causes of upper gastrointestinal bleeding, respectively.

Gastric and duodenal ulcers were detected in 396 of 584 patients. According to the Forrest classification for ulcers, 61 (15.4\%) had type Ia; 73 (18.5\%) had Ib; 184 (46.5\%) had IIa; $72(18.1 \%)$ had IIb and 6 (1.5\%) had IIc.
Table 1. Demographics of the patients

\begin{tabular}{|c|c|}
\hline Age, mean (SD) & $66.36(17.63)$ \\
\hline Male, n (\%) & $373(63.9)$ \\
\hline Medication, n (\%) & $58(9.9)$ \\
\hline Antiplatelets & $43(7.3)$ \\
\hline NSAIDs & $8(1.4)$ \\
\hline Anticoagulants & $7(1.2)$ \\
\hline Melena, n (\%) & $220(37.7)$ \\
\hline Syncope, n (\%) & $359(61.5)$ \\
\hline CLD, n (\%) & $48(8.2)$ \\
\hline \multicolumn{2}{|l|}{ Cause of UGIB } \\
\hline Duodenal Ulcer, n (\%) & $256(43.8)$ \\
\hline Gastric ulcer, n (\%) & $140(24)$ \\
\hline Esophageal varices, $n$ (\%) & $55(9.4)$ \\
\hline Esophageal ulcer, n (\%) & $53(9.1)$ \\
\hline Erosive gastritis ,n (\%) & $21(3.6)$ \\
\hline Malignancy, n (\%) & $19(3.3)$ \\
\hline Others, n (\%) & $40(6.8)$ \\
\hline Hemoglobin, mean (SD) (g/dl) & $9.35(2.3)$ \\
\hline Hematocrites (\%) & $28.4(6.7)$ \\
\hline Platelets (10') & $234(95)$ \\
\hline MPV (fl) & $9.5(1.8)$ \\
\hline PCT & $0.22(0.09)$ \\
\hline PDW (fl) & $13.8(3.3)$ \\
\hline RDW (\%) & $15.5(2.7)$ \\
\hline $1($ RDW $<12.8)$ & $48(8.2)$ \\
\hline $2($ RDW $12.9-14.4)$ & $189(32.4)$ \\
\hline 3 (RDW $14.5-16.5)$ & $195(33.4)$ \\
\hline $4(\mathrm{RDW}>16.5)$ & $152(26)$ \\
\hline \multicolumn{2}{|l|}{ Forrest Classification (n: 396) } \\
\hline Ia, n (\%) & $61(15.4)$ \\
\hline Ib, n (\%) & $73(18.5)$ \\
\hline IIa, n (\%) & $184(46.5)$ \\
\hline IIb, n (\%) & $72(18.1)$ \\
\hline IIc, n (\%) & $6(1.5)$ \\
\hline
\end{tabular}

SD: Standard deviation, n: number, NSAID: Non-steroid anti-inflammatory drugs, CLD: Chronic liver disease, UGIB: Upper gastrointestinal bleeding, MPV: Mean platelet volüme, PCT: Platecrite, PDW: Platelet distrubution width, RDW: Reticulocytes distrubution width

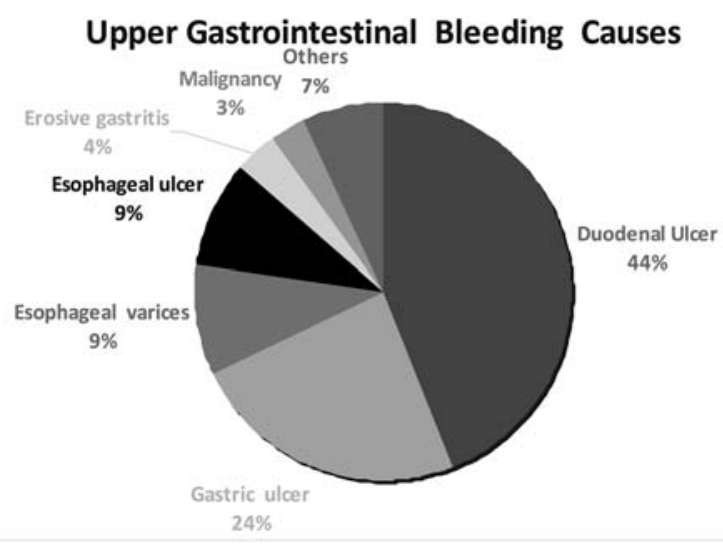

Figure 1 
Table 2. Comparisons of whole blood test parameters between Forrest 1 and Forrest 2 patients

\begin{tabular}{|c|c|c|c|}
\hline & Forrest I & Forrest II & P value \\
\hline Age & $67.7(18)$ & $63.5(18)$ & NS \\
\hline Hemoglobin, mean (SD) (g/dl) & $9.37(2.5)$ & $9.21(2.28)$ & NS \\
\hline Hematocrites, mean (SD) (\%) & $28.4(7.4)$ & $27.9(6.6)$ & NS \\
\hline Platelets, mean (SD) (10 $\left.{ }^{9}\right)$ & $235(79)$ & $243(84)$ & NS \\
\hline MPV, mean (SD) (fl) & $9.5(1.6)$ & $9.5(1.8)$ & NS \\
\hline PCT, mean (SD) & $0.23(0.1)$ & $0.23(0.08)$ & NS \\
\hline PDW, mean (SD) (fl) & $13.5(3.5)$ & $13.9(3)$ & NS \\
\hline RDW, mean (SD) & $15.3(3.3)$ & $15.2(2.5)$ & NS \\
\hline $1(\mathrm{RDW}<12.8), \mathrm{n}(\%)$ & $14(10.4)$ & $23(8.8)$ & NS \\
\hline $2($ RDW $12.9-14.4)$, n $(\%)$ & $52(38.8)$ & $98(37.4)$ & NS \\
\hline 3 (RDW 14.5 - 16.5), n (\%) & $44(32.8)$ & $83(31.7)$ & NS \\
\hline $4(\mathrm{RDW}>16.5), \mathrm{n}(\%)$ & $24(17.9)$ & $58(22.1)$ & NS \\
\hline
\end{tabular}

SD: Standard deviation, n: number, MPV: Mean platelet volume, PCT: Platecrite, PDW: Platelet distrubution width, RDW: Reticulocytes distrubution width

Table 3. Univariable and multivariable logical regression analysis of factors for ulcer diagnosed patients

\begin{tabular}{|l|c|c|c|c|c|c|c|c|}
\hline \multirow{2}{*}{} & \multicolumn{4}{|c|}{ Univariate } & \multicolumn{4}{c|}{ Multivariate } \\
\cline { 2 - 9 } & OR & $\begin{array}{c}\text { Confidence Interval } \\
\text { Lower Upper }\end{array}$ & P value & OR & \multicolumn{2}{c|}{$\begin{array}{c}\text { Confidence Interval } \\
\text { Lower Upper }\end{array}$} & P value \\
\hline Fge & 1,014 & 1,001 & 1,026 & 0,028 & 1,016 & 1,002 & 1,031 & 0,030 \\
\hline Hemale & 1,028 & 0,829 & 1,276 & 0,800 & 0,997 & 0,784 & 1,268 & 0,980 \\
\hline RDW_(1) & Baseline & - & - & 0,775 & -- & - & - & 0,800 \\
\hline RDW_(2) & 1,028 & 0,734 & 1,441 & 0,870 & 1,005 & 0,701 & 1,440 & 0,979 \\
\hline RDW_(3) & 1,028 & 0,723 & 1,461 & 0,880 & 0,983 & 0,669 & 1,442 & 0,928 \\
\hline RDW_(4) & 0,802 & 0,531 & 1,212 & 0,295 & 0,784 & 0,474 & 1,297 & 0,343 \\
\hline Hematocrites & 1,010 & 0,980 & 1,041 & 0,521 & 0,982 & 0,821 & 1,176 & 0,846 \\
\hline PCT & 0,575 & 0,050 & 6,630 & 0,658 & 1,406 & 0,043 & 45,966 & 0,848 \\
\hline MPV & 1,012 & 0,899 & 1,138 & 0,847 & 0,988 & 0,833 & 1,172 & 0,893 \\
\hline PDW & 0,969 & 0,908 & 1,034 & 0,340 & 0,971 & 0,895 & 1,053 & 0,478 \\
\hline PLT & 0,999 & 0,996 & 1,001 & 0,361 & 0,998 & 0,994 & 1,002 & 0,291 \\
\hline Urea & 1,001 & 0,996 & 1,005 & 0,677 & 1,000 & 0,994 & 1,005 & 0,875 \\
\hline Melena & 1,048 & 0,845 & 1,300 & 0,670 & 1,022 & 0,790 & 1,323 & 0,866 \\
\hline Syncope & 0,840 & 0,680 & 1,037 & 0,105 & 0,800 & 0,616 & 1,039 & 0,095 \\
\hline Druguse & 1,326 & 0,942 & 1,866 & 0,105 & 1,140 & 0,773 & 1,680 & 0,509 \\
\hline CLD & 3,181 & 1,082 & 9,354 & 0,036 & 3,431 & 1,145 & 10,280 & 0,028 \\
\hline HF & 2,000 & 0,850 & 4,704 & 0,112 & 1,947 & 0,774 & 4,899 & 0,157 \\
\hline
\end{tabular}

OR: Odds ratio, RDW: Reticulocytes distrubution width, PCT: Platecrite, MPV: Mean platelet volume, PDW: Platelet distrubution width, PLT: Platelets, CLD: Chronic liver disease, HF: Heart Failure

There were no differences in blood cell characteristics (RDW subgroups, PCT, MPV, PDW) or demographics between patients in Forrest I and Forrest II groups (Table 2).
Results of univariable and multivariable logistic regression analysis of factors associated with ulcer diagnosis are presented in Table 3. Only advanced age and presence of CLD were related to re-bleeding risk. 
Table 4. Statistically significant results in comparisons of baseline blood tests and patient demographics among the four RDW groups

\begin{tabular}{|l|c|c|c|c|c|}
\hline & RDW 1 (n:37) & RDW 2 (n:150) & RDW 3 (n:127) & RDW 4 (n:82) & P Value \\
\hline Age, mean, SD & $50.32 \pm 22.77$ & $63.27 \pm 16.94$ & $68.45 \pm 17.36$ & $69.28 \pm 15.29$ & $<0.001$ \\
\hline Hemoglobin, mean, SD & $10.75 \pm 2.18$ & $9.66 \pm 2.17$ & $8.88 \pm 2.41$ & $8.46 \pm 2.25$ & $<0.001$ \\
\hline Hematocrite, mean, SD & $32.12 \pm 6.32$ & $29.05 \pm 6.52$ & $26.95 \pm 7.13$ & $26.34 \pm 6.53$ & $<0.001$ \\
\hline MPV, mean, SD & $10.56 \pm 0.78$ & $10.00 \pm 1.39$ & $9.17 \pm 1.93$ & $8.91 \pm 2.05$ & $<0.001$ \\
\hline PDW, mean, SD & $12.29 \pm 1.93$ & $12.92 \pm 2.90$ & $14.31 \pm 3.13$ & $15.27 \pm 3.56$ & $<0.001$ \\
\hline PLT, mean, SD & $216.38 \pm 55.14$ & $232.05 \pm 66.70$ & $243.18 \pm 87.80$ & $265.89 \pm 105.04$ & 0.006 \\
\hline Urea, mean, SD & $65.81 \pm 34.28$ & $80.99 \pm 39.95$ & $92.58 \pm 50.77$ & $87.87 \pm 49.89$ & 0.009 \\
\hline
\end{tabular}

RDW 1 group $(\mathrm{RDW}<12.8 \%$ ); RDW 2 group (RDW 12.9-14.4\%); RDW 3 group (RDW 14.5-16.5\%); RDW 4 group (RDW > $16.6 \%)$;

RDW: Reticulocytes distrubution width, SD: Standard deviation, MPV: Mean platelet volüme, PDW: Platelet distrubution width, PLT: Platelets

Comparisons of baseline blood tests and patient demographic characteristics among the four RDW groups are presented in Table 4. Haemoglobin, haematocrit and MPV were lower in the high RDW groups (3 and 4) than the low RDW groups (1 and 2). Urea level, platelet count, PDW and mean age were higher in the high RDW groups ( 3 and 4 ) than the low RDW groups (1 and $2 ; \mathrm{P}<.05)$.

\section{DISCUSSION}

AUGIB accounts for a significant portion of emergency department visits and is a cause of serious mortality and morbidity. In this study, duodenal ulcer, gastric ulcer, and oesophageal variceal bleeding were the most common causes of gastrointestinal bleeding. Early diagnosisof AUGIB and early risk stratification improve prognosis. Early identification and treatment of patients who require endoscopy is helpful. Many factors affect severity of gastrointestinal bleeding and re-bleeding risk (7-10).

Recent studies suggest possible biomarkers for determining gastrointestinal bleeding severity. In a study of paediatric patients with Henoch-Schönlein purpura, MPV levels were low among patients with UGIB (15). When acute blood loss occurs, MPV is expected to decrease, whereas platelets are expected to increase (16). However, MPV was higher among patients with UGIB compared with a control group of healthy volunteers in a study Tanoğlu et al.;the authors suggest that this change resulted from sympathetic activity related to hypovolemia and hypotension (17). Changes to platelet shape, size, and function may result from platelet activation in response to improved sympathetic activity $(18,19)$.

Variations in erythropoietin level and erythropoietin non-responsiveness are related to RDW levels.
RDW can be seen physiologically in pregnant persons, black persons, and those who exercise heavily $(20,21)$. Various studies suggest that RDW is an independent risk factor for mortality and morbidity among persons with cancer, diabetes, cardiovascular disease, thromboembolic disease, kidney or liver disease, and inflammatory disease (22-25). RDW may also be related to risk associated with percutaneous coronary angiographic intervention and development of cancer following trauma. In major bleeding, venous inflammation and thrombosis mayincrease inflammatory cytokines, which in turn supress erythrocyte maturation. It has been suggested that suppression of erythrocyte maturation increases RDW $(11,13)$.

In this study,endoscopic Forrest classification was used to divide patients into two groups based on endoscopic findings related to gastric or duodenal ulcers. Demographic characteristics and laboratory parameters were compared between groups. In this preliminary exploration of biomarkers potentially associated with bleeding risk, RDW, PLT, PCT, MPV, and PDW levels did not differ by re-bleeding risk. Furthermore, there were no differences in re-bleeding risk by RDW subgroup. Haemoglobin, haematocrit, and MPV were lower in the high RDW groups (3 and 4) than the low RDW groups (1 and 2). However, urea, PLT, PDW, and mean age were higher in the high RDW groups ( 3 and 4) than the low RDW groups (1 and 2).

Our study should be considered in light of its retrospective design that used existing hospital records only.

In conclusion, AUGIB is among the most common reasons for seeking emergency medical services. Early diagnosis and treatment is important for a favourable prognosis. Although UGE is useful in diagnosing and treating these patients, rapid, practical, easy-to-use, and inexpensive biomarkers are needed to determine bleed- 
ing severity. Our study showed that there were no differencesin these blood count parameters among patients with gastrointestinal ulcers who were at high risk and increased risk of re-bleeding. Future, prospective, randomized controlled studies should address this question.

\section{Abbreviations}

AUGIB - Acute upper gastrointestinal bleeding

RDW - Red blood cell distribution width

PCT - Plateletcrit

MPV - Mean platelet volume

PDW - Platelet distribution width

\section{Acknowledgement:}

None

Conflict of Interests: The authors declare that there are no conflicts of interest related to this article.

Funding: This research did not receive any specific grant from funding agencies in the public, commercial, or not-for-profit sectors.

\section{Licensing}

This work is licensed under a Creative Commons Attribution 4.0 International (CC BY 4.0) License.

\title{
Sažetak
}

\section{KOEFICIJENT VARIJACIJE DISTRIBUCIJE ERITROCITA I TROMBOCITA KAO PREDIKTORA U UTVRĐIVANJU PROGNOZE KRVARENJA IZ GORNJIH PARTIJA GASTROINTESTINALNOG TRAKTA}

\author{
Abaylı Bahri, ${ }^{1}$ Gencdal Genco ${ }^{2}$ \\ ${ }^{1}$ Cukurova Dr. Askım Tufekci Hospital, Department of Gastroenterology and Hepatology, Adana, Turkey \\ ${ }^{2}$ Istanbul Atasehir Memorial Hospital, Department of Gastroenterology, Istanbul, Turkey
}

Uvod i cilj: Akutno krvarenje iz gornjeg dela gastrointestinalnog trakta (AUGIB) je jedno od najčešćih urgentnih stanja u medicini. Rana detekcija pacijenata, koji su pod rizikom je od velikog benefita u pogledu lečenja i prognoze. Istraživali smo da li je težina ulkusne bolesti povezana sa koeficijentom varijacije distribucije eritrocita (RDW), zapreminskim udelom trombocita (PCT), srednjjom vrednošću trombocita (MPV) i koeficijentom distribucije trombocita (PDW).

Materijal i metode: Svi pacijenti, koji su se javili $\mathrm{u}$ urgentno odeljenje sa AUGIB u periodu od januara 2014. do juna 2014. godine bili su uključeni u studiju. Endoskopski nalazi, kompletna krvna slika, demografske karakteristike pacijenata, i endoskopski rezultati su bili sakupljani retrospektivno iz bolničke dokumentacije. Pacijenti sa stadijumom I ili stadijumom II ulkusa

\section{REFERENCES}

1. Barkun A, Bardou M, Marshall JK; Nonvariceal upper GI bleeding consensus conference group. Consensus recommendations for managing patients with nonvariceal upper gastrointestinal bleeding. Ann Intern Med. 2003; 139(10): 843-57.

2. Barkun AN, Bardou M, Kuipers EJ, Sung J, Hunt RH, Martel M, et al. International Consensus Upper Gastrointestinal Bleeding Conference Group. International consensus recommendations on the management of patients with nonvariceal upper gastrointestinal bleeding. Ann Intern Med. 2010; 152(2): 101-13.

3. Hwang JH, Fisher DA, Ben-Menachem T, Chandrasekhara V, Chathadi K, Decker GA, et al. Standards of practice (određivanoj prema Forrest klasifikaciji) bili su uporedivani u pogledu ovih parametara. Rezultati: Ukupno 373 pacijenta i 211 pacijentkinja uzrasta $66,36 \pm 17,36$ godina su uključeni u studiju. Ulkus je bio dijagnostikovan kod 396 od 584 pacijenta (67,8\%). Nije pronađena statistički značajna razlika u pogledu RDW-a ili trombocita između grupa.

Zaključak: Pacijenti koji boluju od AUGIB trebaju biti podvrgnuti urgentnoj dijagnostici i tretmanu. U ovoj studiji pacijenti sa AUGIB, grupisani prema etiologiji krvarenja, verovatni rani prognostički parametri nisu bili povezani sa težinom krvarenja u grupi pacijenata sa dijagnostikovanim gastričnim ili duodenalnim ulkusom.

Ključne reči: endoskopija, gastrointestinalno krvarenje, ulkus, RDW, PCT, MPV, PDW.

committee of the American Society for Gastrointestinal Endoscopy. The role of endoscopy in the management of acute non-variceal upper GI bleeding. Gastrointest Endosc. 2012; 75(6): 1132-8.

4. Gralnek IM, Dumonceau JM, Kuipers EJ, Lanas A, Sanders DS, Kurien M, et al. Diagnosis and management of nonvariceal upper gastrointestinal hemorrhage: European Society of Gastrointestinal Endoscopy (ESGE) Guideline. Endoscopy. 2015; 47(10): a1-46. Epub 2015 Sep 29.

5. Laine L, Peterson WL. Bleeding peptic ulcer. N Engl J Med. 1994; 331(11): 717-27.

6. Holster IL, Kuipers EJ. Management of acute nonvariceal upper gastrointestinal bleeding: current policies and future perspectives. World J Gastroenterol. 2012; 18(11): 1202-7. 
7. Nable JV, Graham AC. Gastrointestinal bleeding. Emerg Med Clin North Am. 2016; 34(2): 309-25.

8. Dabbah S, Hammerman H, Markiewicz W, Aronson D. Relation between red cell distribution width and clinical outcomes after acute myocardial infarction. Am J Cardiol. 2010; 105(3): 312-7.

9. Braun E, Domany E, Kenig Y, Mazor Y, Makhoul BF, Azzam ZS. Elevated red cell distribution width predicts poor outcome in young patients with community acquired pneumonia. Crit Care. 2011; 15(4): R194.

10. Hampole CV, Mehrotra AK, Thenappan T, Gomberg-Maitland M, Shah SJ. Usefulness of red cell distribution width as a prognostic marker in pulmonary hypertension. Am J Cardiol. 2009; 104(6): 868-72.

11. Fatemi O, Torguson R, Chen F, Ahmad S, Badr S, Satler LF, et al. Red cell distribution width as a bleeding predictor after percutaneous coronary intervention. Am Heart J. 2013; 166(1): 104-9.

12. Altintas O, Duruyen H, Baran G, Baran O, Katar S, Antar V, et al. The relationship of hematoma growth to red blood cell distribution width in patients with hypertensive intracerebral hemorrhage. Turk Neurosurg. 2017; 27(3): 368-73.

13. Paulus EM, Weinberg JA, Magnotti Lj, Sharpe JP, Schroeppel TJ, Fabian TC, et al. Admission red cell distribution width: a novel predictor of massive transfusion after injury. Am Surg. 2014; 80(7): 685-9.

14. Forrest JA, Finlayson ND, Shearman DJ. Endoscopy in gastrointestinal bleeding. Lancet. 1974; 2(7877): 394-7.

15. Makay B, Türky1lmaz Z, Duman M, Ünsal E. Mean platelet volume in Henoch-Schönlein purpura: relationship to gastrointestinal bleeding. Clin Rheumatol. 2009; 28(10): 1225-8.

16. Giles C. The platelet count and mean platelet volume. Br J Haematol. 1981; 48(1): 31-7.

\author{
Correspondence to/Autor za korespondenciju \\ Genco Gençdal \\ Address: Istanbul Atasehir Memorial Hospital, \\ Department of Gastroenterology, GOP, Istanbul, Turkey \\ Phone Number: 090-0216-5706666 090-5063581760 \\ E-mail: gencogencdal@yahoo.co.uk \\ Fax Number: 090-216-5706620
}

17. Tanoglu A, Eroglu M, Osman Yildirim AO, Kara M, Yazgan Y. Increased Mean Platelet Volume Is Associated With Duration Of Hospitalization And Transfusion Requirement In Upper Gastrointestinal Bleedings. Gulhane Med J. 2015; 57(1): 16-20.

18. Thompson CB, Eaton KA, Princiotta SM, Rushin CA, Valeri CR. Size dependent platelet subpopulations: relationship of platelet volume to ultrastructure, enzymatic activity, and function. Br J Haematol. 1982; 50(3): 509-19.

19. Hjemdahl P, Larsson PT, Wallén NH. Effects of stress and beta-blockade on platelet function. Circulation. 1991; 84(suppl 6): VI44-61.

20. Kario K, Matsuo T, Nakao K, Yamaguchi N. The correlation between red cell distribution width and serum erythropoietin titres. Clin Lab Haematol. 1991; 13(2): 222-3.

21. Afsar B, Saglam M, Yuceturk C, Agca E. The relationship between red cell distribution width with erythropoietin resistance in iron replete hemodialysis patients. Eur J Intern Med. 2013; 24(3): e25-9.

22. Salvagno GL, Sanchis-Gomar F, Picanza A, Lippi G. Red blood cell distribution width: A simple parameter with multiple clinical applications. Crit Rev Clin Lab Sci. 2015; 52(2): 86-105.

23. Dabbah S, Hammerman H, Markiewicz W, Aronson D. Relation between red cell distribution width and clinical outcomes after acute myocardial infarction. Am J Cardiol. 2010; 105(3): 312-7.

24. Braun E, Domany E, Kenig Y, Mazor Y, Makhoul BF, Azzam ZS. Elevated red cell distribution width predicts poor outcome in young patients with community acquired pneumonia. Crit Care. 2011; 15(4): R194.

25. Patel KV, Ferrucci L, Ershler WB, Longo DL, Guralnik JM. Red blood cell distribution width and the risk of death in middle-aged and older adults. Arch Intern Med. 2009; 169(5): 515-23. 